\title{
Prevalência da Incontinência Urinária em Mulheres Atletas Praticantes de Esporte de Alto Impacto: Uma Revisão Integrativa
}

\author{
Zenilda Santos Silva ${ }^{1}$ : Lucimauro Silva Palles ${ }^{2}$; Juliana Barros Ferreira ${ }^{3}$
}

\begin{abstract}
Resumo: A IU pode afetar quase $80 \%$ das mulheres que praticam alguma modalidade esportiva, bem como enquanto realiza exercícios de alta intensidade. Objetivo: realizar uma revisão integrativa para investigar a prevalência da incontinência urinária em mulheres atletas praticantes de esportes de alto impacto para contribuir com a tomada de medidas preventivas que minimizem os impactos negativos da incontinência urinária. Métodos: revisão integrativa da literatura, de acordo com a metodologia PICO (participantes, intervenção, contexto e resultados), cuja coleta de dados foi realizada nas bases de dados: BIREME, MEDLINE, LILACS, SciELO, PUBMED e PEDro, no mês de setembro de 2020. A amostra foi composta por 11 artigos publicados entre nos anos de 2010 a 2020 em periódicos nacionais e internacionais. Resultados: os estudos que compõem essa revisão integrativa mostraram que a prática dessas modalidades esportivas pode ser causadora da Incontinência Urinária, embora não haja especificação do tipo, intensidade e comprometimento dessa prática pela presença da Incontinência Urinária. Conclusão: A incontinência urinária que pode ser causada pela prática de esportes de alto impacto em mulheres, porém nem sempre a IU está diretamente relacionada com a prática esportiva de alto impacto, e que a presença de outros fatores pode fazer emergir a incontinência urinária.
\end{abstract}

Palavras-chave: Incontinência urinária, esportes de alto impacto, revisão integrativa;

\section{Prevalence of Urinary Incontinence in Female Athletes Practicing High Impact Sports: An Integrative Review}

\begin{abstract}
UI can affect almost $80 \%$ of women who practice some sport, as well as while performing high intensity exercises. OBJECTIVE: to conduct an integrative review to investigate the prevalence of urinary incontinence in female athletes practicing high-impact sports to contribute to preventive measures that minimize the negative impacts of urinary incontinence. Methods: integrative literature review, according to the PICO methodology (participants, intervention, context and results), whose data collection was performed in the following databases: BIREME, MEDLINE, LILACS, SciELO, PUBMED and PEDro, in September 2020. The sample consisted of 11 articles published between 2010 and 2020 in national and international journals. Results: the studies that make up this integrative review showed that the practice of these sports can cause Urinary Incontinence, although there is no specification of the type, intensity and impairment of this practice due to the presence of Urinary Incontinence. Conclusion: Urinary incontinence, which can be caused by the practice of high-impact sports in women, but UI is not always directly related to high-impact sports, and that the presence of other factors can cause urinary incontinence to emerge.
\end{abstract}

Keywords: Urinary incontinence, high impact sports, integrative review;

\footnotetext{
${ }^{1}$ Graduanda em Fisioterapia pela Uninassau - Vitória da Conquista, e-mail: nildafisioterapeuta@gmail.com.

${ }^{2}$ Psicólogo, Especialista em Neuropsicologia, Mestre em Linguística (UESB), e-mail: maupalles@gmail.com.

${ }^{3}$ Fisioterapeuta, Mestre em Tecnologias em Saúde/EBMSP. Docente da Maurício de Nassau em Vitória da Conquista/BA, e-mail: julibarros78@hotmail.com.
} 


\section{Introdução}

A incontinência urinária (IU) pode ser definida como uma perda de urina de forma involuntária, segundo a Sociedade Internacional de Continência (ICS), essa condição pode fazer surgir complicações para a saúde tanto do ponto de vista médico, social, econômico quanto psicológico, e afeta de forma negativa a qualidade de vida das pessoas, de forma mais grave nas mulheres (PRIGOL, SEBBEN e GUEDES, 2014).

A IU feminina pode ter relações com a anatomia da pelve, hormônios, cirurgias de partos e gestações que podem se deslocar e contribuir no enfraquecimento dos músculos períneos que são compostos por um conjunto de partes moles que fecham a pelve e suportam as vísceras em posição vertical (SANTOS, 2018). Fatores a exemplo da idade, menopausa, obesidade, cirurgias ginecológicas, doenças crônicas, fatores hereditários, uso de drogas, constipação intestinal, consumo de cafeína, consumo de cigarro e prática de exercícios físicos podem ser considerados de risco impactarem no aumento da perda urinária (SANTOS, 2018).

A literatura afirma que IU pode afetar quase $80 \%$ das mulheres que pratica alguma modalidade esportiva, bem como enquanto realiza exercícios de alta intensidade (ALVES et al., 2017; BARRETO et al., 2014; BO, K., 2010; HAVGOSKA et al., 2017; MACHADO, 2017; PEREIRA et al., 2012). Destaca-se que nem sempre o profissional de Educação Física, que conduz e orienta os treinos, dispõe de conhecimento teórico sobre a saúde da mulher, como o assoalho pélvico, que necessita de cuidados diferenciados (ROSA, VARGAS, KELLER, LIMA, KLAHR e ROSA, 2016).

O percentual de mulheres que praticam exercícios de alta intensidade está entre $28 \%$ a $68 \%$, vale ressaltar que essa prática pode fazer emergir a incontinência urinária de esforço (IUE) (BO, K., 2010; ALVES et al., 2017). Esta disfunção ocorre por meio do aumento da pressão intra abdominal provocando sobrecarga nos músculos pélvicos (ALVES et al., 2017; ARAUJO, et al., 2008; BARRETO et al., 2014; BO, K. 2010). Um estudo com jogadoras de futebol, realizado por Fernandes et al. (2015), foi encontrado presença de IU em jogadoras futebol, e também uma revisão sistemática mostrou que a IU durante a prática da atividade física é um evento comum (NYGAARD et al, 2016).

A partir desses dados, e pelo aumento da prática de exercícios, e atividades de alto impacto pela população feminina, objetiva-se assim realizar uma revisão integrativa para investigar a prevalência da incontinência urinária em mulheres atletas praticantes de esportes 
de alto impacto para contribuir com a tomada de medidas preventivas que minimizem os impactos negativos da incontinência urinária.

\section{Metodologia}

Este estudo é composto por uma revisão integrativa da literatura sobre a presença da incontinência urinária em mulheres atletas praticantes de esportes de alto impacto. Esse tipo de estudo busca, avalia e apresenta uma síntese dos dados coletados sobre um tema específico. Como resultados apresenta-se a possibilidade de uma investigação científica com vistas a contribuir na implementação de políticas públicas de saúde para o atendimento à mulher, reduzindo custos e direcionamento para estudos a serem realizados com essa temática (TEIXEIRA; MEDEIROS; NASCIMENTO; SILVA; RODRIGUES; 2013).

Na elaboração desta revisão integrativa, formaram-se as seguintes questões seguindo a estratégia PICO (P - população; I - intervenção/área de interesse; C - Comparação; O desfecho). Nesse sentido, considera-se: $\mathrm{P}$ - mulheres atletas praticantes de esportes de alto impacto, I - incontinência urinária, C - Sem comparação, O - modalidades esportivas que provocam maior comprometimento de incontinência urinária. Assim, formulou-se a seguinte pergunta de investigação: Qual a prevalência da incontinência urinária em mulheres atletas praticantes de esporte de alto impacto?

O desenho desse estudo seguiu o método composto por seis etapas diferentes e complementares: a) elaboração da questão (problema) de pesquisa; b) seleção da amostra com os descritores temáticos; c) coleta de dados em bases científicas; d) avaliação dos dados coletados referentes à temática; e) análise e interpretação dos dados encontrados; e, f) publicação dos dados. A busca das publicações foi realizada em setembro 2020, em partir dos artigos publicados na BIREME, Medical Literature Analysis and Retrieval System (MEDLINE), Literatura Latino-Americana e do Caribe em Ciências da Saúde (LILACS), Scientific Electronic Library Online (SciELO), serviço da U. S. National Library of Medicine (NLM) PUBMED, e Physiotherapy Evidence Database (PEDro).

Foram definidos os descritores contidos nos Descritores em Ciências da Saúde (DECS) pesquisados nas bases: "incontinência urinária", "mulheres atletas”, “esportes alto impacto", "women athletes" "playing" "high impact sports". Esses descritores foram pesquisados nos idiomas inglês e português do Brasil. 
Os descritores foram utilizados em pesquisas que os contivessem no título, resumo e assunto. Na estratégia de combinação dos descritores, utilizou-se o termo boleano "AND” nas seguintes combinações: "women athletes" AND “playing” AND "high impact sports".

Como critério de inclusão foram alocados artigos que tratam sobre incontinência urinária em atletas praticantes de esportes de alto impacto, publicações em periódicos científicos nacionais e internacionais no período de 10 anos, entre os anos de 2010-2020. Como critério de exclusão, foram descartados artigos nos formatos de revisão, resenhas, dissertações, monografias, teses e resumos em anais de eventos. As publicações selecionadas formam um quadro organizado (quadro 1) por tema com ordenamento e avaliação da aproximação e concordância com a investigação temática desse estudo.

Conforme os critérios de inclusão e exclusão, foi realizada a leitura dos títulos e resumos dos artigos científicos para certificar sua adequação e possibilidade de responder à questão norteadora da presente investigação. O fluxograma, (Figura 1), descreve o percurso de identificação, seleção e inclusão dos estudos primários selecionados, segundo as bases de dados, critérios de inclusão e exclusão e leitura de título e resumo totalizando os 11 (onze) artigos selecionados para a redação desta revisão integrativa.

Figura 1: Fluxograma da pesquisa em Artigos Científicos

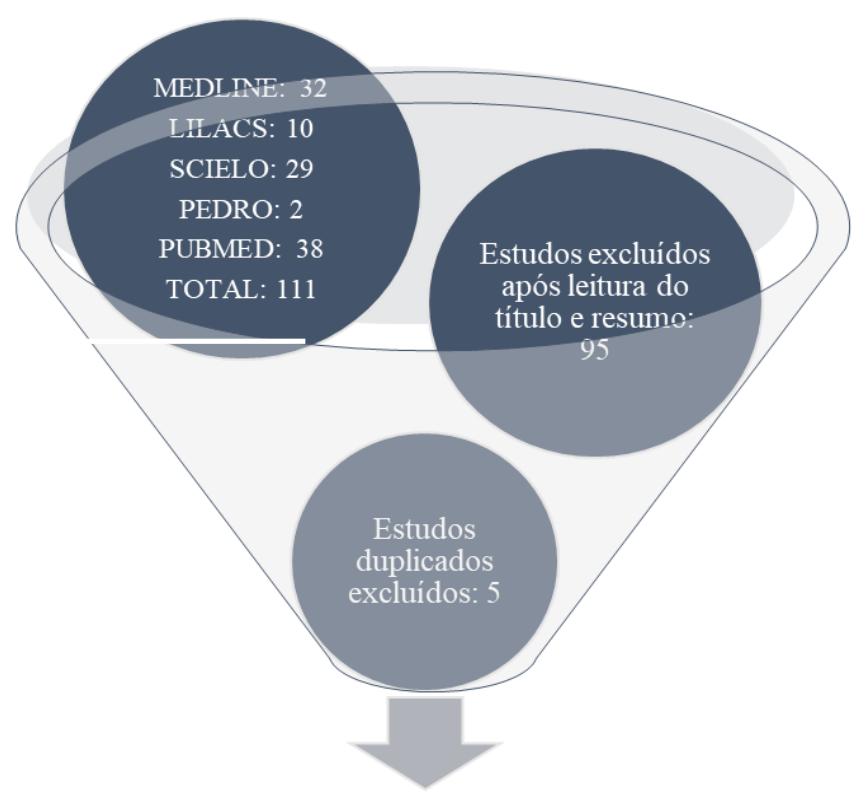

Estudos primários incluídos para análise: 11

Fonte: Dados da pesquisa 


\section{Resultados}

Dos 11 artigos selecionados para a composição desta revisão integrativa de literatura, 03 (três) foram publicados no ano de 2012, 01 (um) artigo em 2014, 02 (dois) artigos publicados em 2018, no ano de 2019 foram publicados 02 (dois) artigos, e em 2020, foi encontra 01 (uma) publicação. Quando se refere ao local das pesquisas, a maior parte dos estudos foram brasileiros, 09 (nove) pesquisas, equivalente a $81 \%, 02$ (dois) estudos, correspondente a 19\% são estudos internacionais realizados nos países da Noruega e da República Eslovaca. Dessa forma, a evidência científica predominante tem hierarquia 2 que são estudos de evidência bem delineados sem randomização, coorte ou caso-controle, todos de delineamento transversal. Outro aspecto relevante está nas modalidades esportivas que podem apresentar a prevalência da Incontinência urinária: voleibol, ginástica rítmica, exercícios físicos praticados em academias, musculação, crossfit, corrida de rua, jump e pilates. Os resultados são apresentados nos Quadro 1 que apresenta os dados editorais e o Quadro 2 que mostra dois aspectos de análise: objetivos e resultados, que foram base para a seleção nesse estudo, a numeração atribuída aos artigos foi aleatória.

Quadro 1 - Caracterização dos artigos selecionados com os dados editorais: autor(es), ano, local, delineamento e nível de evidência.

\begin{tabular}{|c|l|l|c|c|c|c|}
\hline No & \multicolumn{1}{|c|}{ Título } & \multicolumn{1}{c|}{ Autor(es) } & Ano & Local & Delineamento & $\begin{array}{c}\text { Nível de } \\
\text { Evidência }\end{array}$ \\
\hline A01 & $\begin{array}{l}\text { Prevalence and risk of } \\
\text { sport types to stress } \\
\text { urinary incontinence in } \\
\text { sportswomen: A cross- } \\
\text { sectional study }\end{array}$ & $\begin{array}{l}\text { Magdaléna Hagovska } \\
\text { Ján Švihra } \\
\text { Alena Buková } \\
\text { Dana Dračková } \\
\text { Viera Švihrová }\end{array}$ & 2018 & $\begin{array}{l}\text { República } \\
\text { Eslovaca }\end{array}$ & Transversal & 2 \\
\hline $\begin{array}{l}\text { A02 } \\
\text { gymnasts and urinary } \\
\text { incontinence: } \\
\text { Prevalence, risk } \\
\text { factors, and influence } \\
\text { on performance }\end{array}$ & $\begin{array}{l}\text { Karte C. D. Gram } \\
\text { Kari Bø }\end{array}$ & 2020 & Noruega & Transversal & 2 \\
\hline A03 & $\begin{array}{l}\text { Disfunções urinárias } \\
\text { em mulheres } \\
\text { praticantes de } \\
\text { atividade física em } \\
\text { academias - um estudo } \\
\text { transversal }\end{array}$ & $\begin{array}{l}\text { Leidiany B. da Silva } \\
\text { Wyrla O. Santos } \\
\text { Nazete dos S. } \\
\text { Araújo, Cibele N. C. } \\
\text { Rodrigues } \\
\text { Erica F. C. Nunes }\end{array}$ & 2018 & Brasil & Transversal & 2 \\
\hline
\end{tabular}




\begin{tabular}{|c|c|c|c|c|c|c|}
\hline A04 & $\begin{array}{l}\text { Prevalência de } \\
\text { Incontinência Urinária } \\
\text { em Mulheres } \\
\text { Praticantes de Pilates e } \\
\text { de Musculação }\end{array}$ & $\begin{array}{l}\text { Líllian F. de Amorim } \\
\text { Daiane S. D. Saraiva } \\
\text { Rosana P. Cirqueira }\end{array}$ & 2019 & Brasil & Transversal & 2 \\
\hline A05 & $\begin{array}{l}\text { Prevalência de } \\
\text { incontinência urinária } \\
\text { e perfil miccional de } \\
\text { mulheres praticantes } \\
\text { de } \\
\text { Crossfit }{ }^{\circledR}\end{array}$ & $\begin{array}{l}\text { Larissa de O. Leal } \\
\text { Mariana Alves } \\
\text { Santos } \\
\text { Nilce M. de F. } \\
\text { Santos } \\
\text { Lays M. Braga } \\
\text { Kelly C. de Faria } \\
\text { Nunes }\end{array}$ & 2018 & Brasil & Transversal & 2 \\
\hline A06 & $\begin{array}{l}\text { Incontinência urinária } \\
\text { em mulheres jovens } \\
\text { praticantes de } \\
\text { exercício físico }\end{array}$ & $\begin{array}{l}\text { Lislei J. Patrizzi } \\
\text { Dayane A. Viana } \\
\text { Lilane M. A. Silva } \\
\text { Maycon S. Pegorari }\end{array}$ & 2014 & Brasil & Transversal & 2 \\
\hline A07 & $\begin{array}{l}\text { Prevalência de } \\
\text { incontinência urinária } \\
\text { em atletas praticantes } \\
\text { de corrida de rua }\end{array}$ & $\begin{array}{l}\text { Franciely da R. } \\
\text { Ferraz } \\
\text { Adriana A. Ferraz } \\
\text { Erica F. C. Nunes } \\
\text { Gustavo F. Sutter } \\
\text { Latorre }\end{array}$ & 2012 & Brasil & Transversal & 2 \\
\hline A08 & $\begin{array}{l}\text { A prevalência de } \\
\text { incontinência urinária } \\
\text { em mulheres } \\
\text { praticantes de jump }\end{array}$ & $\begin{array}{l}\text { Priscilla P. de } \\
\text { Almeida } \\
\text { Lívia R. G. Machado }\end{array}$ & 2012 & Brasil & Transversal & 2 \\
\hline A09 & $\begin{array}{l}\text { Incontinência urinária } \\
\text { de esforço em } \\
\text { mulheres praticantes } \\
\text { de crossfitß: Um } \\
\text { estudo transversal de } \\
\text { prevalência }\end{array}$ & $\begin{array}{l}\text { Monique Bogéa } \\
\text { Priscila Gomes } \\
\text { Ana Dinucci } \\
\text { Fábio Dutra } \\
\text { Patrícia Zaidan }\end{array}$ & 2019 & Brasil & Transversal & 2 \\
\hline A10 & $\begin{array}{l}\text { Prevalência de } \\
\text { incontinência urinária } \\
\text { em mulheres do curso } \\
\text { de educação física } \\
\text { da Universidade do } \\
\text { Sul de Santa Catarina, } \\
\text { campus Tubarão }\end{array}$ & $\begin{array}{l}\text { Luiz A. Pensin, } \\
\text { Gregório W. Pereira } \\
\text { Juliano D. Fransozi } \\
\text { Lucas Pensin } \\
\text { Soraya Lamim Bello } \\
\text { Sandro P. Berni } \\
\text { Brum }\end{array}$ & 2012 & Brasil & Transversal & 2 \\
\hline A11 & $\begin{array}{l}\text { Prevalência de } \\
\text { incontinência urinária } \\
\text { de esforço em } \\
\text { mulheres praticantes } \\
\text { de crossfit }\end{array}$ & $\begin{array}{l}\text { Letícia Ferreira } \\
\text { Vitória Laura de } \\
\text { Melo } \\
\text { Mirella Dias }\end{array}$ & 2019 & Brasil & Transversal & 2 \\
\hline
\end{tabular}


Quadro 2 - Caracterização dos artigos selecionados com seus objetivos e resultados.

\begin{tabular}{|c|c|c|c|}
\hline $\mathbf{N}^{\circ}$ & Título & Objetivos & Resultados \\
\hline A01 & $\begin{array}{l}\text { Prevalence and risk } \\
\text { of sport types to } \\
\text { stress urinary } \\
\text { incontinence in } \\
\text { sportswomen: A } \\
\text { cross-sectional study }\end{array}$ & $\begin{array}{l}\text { Investigar a prevalência } \\
\text { e o risco de desenvolver } \\
\text { incontinência urinária } \\
\text { por estresse (SUI) em } \\
\text { cada tipo de esporte de } \\
\text { alta intensidade e o } \\
\text { impacto associado à } \\
\text { qualidade de vida das } \\
\text { esportistas. }\end{array}$ & $\begin{array}{l}\text { As jogadoras de voleibol têm } 116 \% \\
\text { de chance de adquirir IUE em } \\
\text { comparação com as mulheres que } \\
\text { praticam outras modalidades } \\
\text { esportivas analisadas neste estudo. } \\
\text { Os profissionais de saúde devem } \\
\text { informar a população de esportistas } \\
\text { com fatores de risco para IUE, a fim } \\
\text { de implementar fisioterapia } \\
\text { preventiva para o fortalecimento da } \\
\text { musculatura do assoalho pélvico. }\end{array}$ \\
\hline A02 & $\begin{array}{l}\text { High level rhythmic } \\
\text { gymnasts and urinary } \\
\text { incontinence: } \\
\text { Prevalence, risk } \\
\text { factors, and influence } \\
\text { on performance }\end{array}$ & $\begin{array}{l}\text { Investigar a prevalência } \\
\text { e os fatores de risco para } \\
\text { IU em ginastas rítmicas e } \\
\text { o impacto da IU no } \\
\text { desempenho. }\end{array}$ & $\begin{array}{l}\text { IMC, hipermobilidade, menarca, } \\
\text { alimentação desordenada e horas de } \\
\text { treinamento não foram considerados } \\
\text { fatores de risco para incontinência } \\
\text { urinária de esforço. Concluindo, a IU } \\
\text { é comum em ginastas rítmicas e pode } \\
\text { influenciar o desempenho esportivo. }\end{array}$ \\
\hline A03 & $\begin{array}{l}\text { Disfunções urinárias } \\
\text { em mulheres } \\
\text { praticantes de } \\
\text { atividade física em } \\
\text { academias - um } \\
\text { estudo transversal }\end{array}$ & $\begin{array}{l}\text { Verificar a prevalência } \\
\text { de IUE em mulheres com } \\
\text { prática regular de } \\
\text { atividade física em } \\
\text { academias. }\end{array}$ & $\begin{array}{l}\text { A prevalência de IU em mulheres } \\
\text { praticantes de exercícios aeróbicos e } \\
\text { musculação nas academias foi baixa } \\
(12 \%) \text {, com severidade de moderada } \\
\text { a grave e sem associação com idade, } \\
\text { modalidade, tempo de prática, turno } \\
\text { e frequência de exercícios }\end{array}$ \\
\hline A04 & $\begin{array}{l}\text { Prevalência de } \\
\text { Incontinência } \\
\text { Urinária em } \\
\text { Mulheres } \\
\text { Praticantes de Pilates } \\
\text { e de Musculação }\end{array}$ & $\begin{array}{l}\text { obter dados } \\
\text { socioeconômicos e } \\
\text { demográficos da } \\
\text { população estudada, } \\
\text { além de coletar } \\
\text { informações referentes à } \\
\text { existência de possíveis } \\
\text { incontinências urinárias, } \\
\text { seja por esforço ou } \\
\text { urgência, em } \\
\text { mulheres jovens ativas, } \\
\text { através do questionário } \\
\text { validado para o } \\
\text { português ICIQ-SF }\end{array}$ & $\begin{array}{l}\text { são necessários mais estudos com } \\
\text { amostragens maiores objetivando } \\
\text { investigar as relações da IU com este } \\
\text { tipo de atividade física. Além disso, } \\
\text { mesmo com um número amostral } \\
\text { pequeno, este trabalho indica que se } \\
\text { tenha mais atenção e cuidado, seja } \\
\text { pelos praticantes ou pelos } \\
\text { profissionais orientadores de } \\
\text { musculação, logo que, a amostra, } \\
\text { mesmo com } 10 \text { anos a menos em } \\
\text { média, quando comparada com a } \\
\text { amostra de pilates, se mostrou mais } \\
\text { suscetível } \\
\text { a IU. }\end{array}$ \\
\hline A05 & $\begin{array}{l}\text { Prevalência de } \\
\text { incontinência } \\
\text { urinária e perfil } \\
\text { miccional de } \\
\text { mulheres praticantes } \\
\text { de } \\
\text { Crossfit }{ }^{\circledR}\end{array}$ & $\begin{array}{l}\text { Avaliar a prevalência de } \\
\text { perda de urina } \\
\text { involuntária e padrão } \\
\text { miccional em mulheres } \\
\text { praticantes de } \\
\text { Crossfit@ em de Patos de } \\
\text { Minas/MG }\end{array}$ & $\begin{array}{l}\text { Observou-se que } 78,9 \% \text { eram } \\
\text { nulíparas, } 13,2 \% \text { tiveram parto do } \\
\text { tipo } \\
\text { vaginal e } 7,9 \% \text { partos cesárea. } \\
\text { Apenas } 7,9 \% \text { da amostra apresentou } \\
\text { incontinência atlética. Conclusão: } \\
\text { Apesar da queixa, houve baixa } \\
\text { prevalência de Incontinência Atlética } \\
\text { na amostra. }\end{array}$ \\
\hline
\end{tabular}




\begin{tabular}{|c|c|c|c|}
\hline A06 & $\begin{array}{l}\text { Incontinência } \\
\text { urinária em mulheres } \\
\text { jovens praticantes de } \\
\text { exercício físico }\end{array}$ & $\begin{array}{l}\text { Comparar a prevalência } \\
\text { do autorrelato de } \\
\text { incontinência urinária em } \\
\text { mulheres jovens de } \\
\text { acordo com as } \\
\text { modalidades de exercício } \\
\text { físico }\end{array}$ & $\begin{array}{l}\text { A IU está presente nas mulheres em } \\
\text { grande percentual nas diferentes } \\
\text { modalidades esportivas, com } \\
\text { associação significativa na } \\
\text { musculação. }\end{array}$ \\
\hline A07 & $\begin{array}{l}\text { Prevalência de } \\
\text { incontinência } \\
\text { urinária em atletas } \\
\text { praticantes de corrida } \\
\text { de rua }\end{array}$ & $\begin{array}{l}\text { Avaliar a prevalência de } \\
\text { IU em atletas praticantes } \\
\text { de corrida de rua }\end{array}$ & $\begin{array}{l}\text { Prevalência em IU condizente com } \\
\text { estudos anteriores, sendo o tipo de IU } \\
\text { mais prevalente foi a IUE o que } \\
\text { reforça a análise de demais autores. } \\
\text { Por meio de nossos achados foi } \\
\text { possível evidenciar que não há um } \\
\text { grande impacto na qualidade de vida } \\
\text { dos atletas. }\end{array}$ \\
\hline A08 & $\begin{array}{l}\text { A prevalência de } \\
\text { incontinência } \\
\text { urinária em mulheres } \\
\text { praticantes de jump }\end{array}$ & $\begin{array}{l}\text { identificar a prevalência } \\
\text { de IU em um grupo de } \\
\text { mulheres praticantes de } \\
\text { jump }\end{array}$ & $\begin{array}{l}\text { Existe incontinência urinária nas } \\
\text { praticantes de jump investigadas, } \\
\text { multíparas e nulíparas, em jovens, } \\
\text { durante a prática de jump e em outras } \\
\text { ocasiões, apontando a necessidade de } \\
\text { estudos mais abrangentes sobre a } \\
\text { temática. }\end{array}$ \\
\hline A09 & $\begin{array}{l}\text { Incontinência } \\
\text { urinária de esforço } \\
\text { em mulheres } \\
\text { praticantes de } \\
\text { crossfit@: } \\
\text { Um estudo } \\
\text { transversal de } \\
\text { prevalência }\end{array}$ & $\begin{array}{l}\text { Verificar a prevalência } \\
\text { de estresse e } \\
\text { incontinência urinária em } \\
\text { mulheres que praticam } \\
\text { Crossfit@ }\end{array}$ & $\begin{array}{l}\text { Os resultados podem sugerir a } \\
\text { possibilidade etiológica do Crossfit }{ }^{\circledR} \\
\text { para incontinência urinária, } \\
\text { entretanto, o intervalo de confiança } \\
\text { apresentado nos obriga a entender } \\
\text { que este resultado aconteceu ao } \\
\text { acaso. No entanto, não podemos } \\
\text { afirmar com firmeza o risco de } \\
\text { Crossfit }{ }^{\circledR} \text { prática para IUE, uma vez } \\
\text { que não foi observada diferença } \\
\text { significativa entre os grupos. }\end{array}$ \\
\hline $\mathbf{A 1 0}$ & $\begin{array}{l}\text { Prevalência de } \\
\text { incontinência } \\
\text { urinária em mulheres } \\
\text { do curso de educação } \\
\text { física } \\
\text { da Universidade do } \\
\text { Sul de Santa } \\
\text { Catarina, campus } \\
\text { Tubarão }\end{array}$ & $\begin{array}{l}\text { Avaliar a prevalência de } \\
\text { incontinência urinária em } \\
\text { mulheres graduandas do } \\
\text { curso de Educação Física } \\
\text { da Universidade do Sul } \\
\text { de Santa Catarina } \\
\text { (UNISUL), campus } \\
\text { Tubarão. }\end{array}$ & $\begin{array}{l}\text { Apresentou uma prevalência de } \\
\text { incontinência urinária significativa e } \\
\text { semelhante a outros estudos } \\
\text { encontrados na literatura nacional e } \\
\text { internacional }\end{array}$ \\
\hline A11 & $\begin{array}{l}\text { Prevalência de } \\
\text { incontinência } \\
\text { urinária de esforço } \\
\text { em mulheres } \\
\text { praticantes de crossfit }\end{array}$ & $\begin{array}{l}\text { Verificar a prevalência } \\
\text { de incontinência urinária } \\
\text { de } \\
\text { esforço em mulheres } \\
\text { praticantes de Crossfit }\end{array}$ & $\begin{array}{l}\text { Nota-se a necessidade de outras } \\
\text { pesquisas com uma amostra mais } \\
\text { ampla, maior homogeneidade de } \\
\text { treinos e individualização na } \\
\text { avaliação. }\end{array}$ \\
\hline
\end{tabular}

Fonte: Dados da pesquisa 


\section{Discussão}

Os estudos que compõem essa revisão integrativa para investigar a prevalência da incontinência urinária em mulheres atletas praticantes de esportes de alto impacto mostrou que a prática dessas modalidades esportivas pode ser causadora da Incontinência Urinária, embora não haja especificação do tipo, intensidade e comprometimento dessa prática pela presença da Incontinência Urinária.

Hagovska, et al., (2018) relata que atletas de voleibol podem ter maiores chances de desenvolverem a incontinência urinária de esforço (IUE) quando se comparam com outras modalidades esportivas. Ainda segundo esses autores os profissionais de saúde devem disseminar as informações, em especial para que profissionais a exemplo de fisioterapeutas que podem realizar prevenção através dos recursos e técnicas da fisioterapia para o fortalecimento da musculatura do assoalho pélvico. Ferraz, et al, (2012) afirmam que a IUE é mais prevalente em atletas de corrida de rua sem impacto na qualidade de vida das atletas dessa modalidade. Para Gram e Bø, (2020) em seu estudo com atletas de ginástica rítmica, apontam que fatores como IMC, hipermobilidade, menarca, alimentação desordenada e horas de treinamento não são considerados fatores de riscos para a IUE, esse estudo ainda apresenta como resultado que a IU pode influenciar no desempenho esportivo.

Conforme Pensin, et. al, (2012), encontraram significativa prevalência de IU em mulheres estudantes de educação física. Discordando desse resultado, Silva, et al, (2018) realizou um estudo com mulheres praticantes de exercícios físicos em academias, e demonstraram que as praticantes de exercícios aeróbicos e musculação foi baixa (12\%), com gravidade moderada e sem associação com aspectos como: idade, modalidade, tempo de prática, turno e frequência de exercícios.

No estudo conduzido por Amorim, et. al (2019) com mulheres praticantes de musculação e pilates, não encontrou dados relevantes para afirmar que existe relação direta entre IU com a prática dessas modalidades, embora haja indícios que as praticantes de pilates tem maior suscetibilidade para desenvolver IU do que as praticantes de musculação, enquanto que Patrizzi (2014) em seu estudo com mulheres praticantes de exercício físico afirma que a IU está presente em diferentes modalidades esportivas com associação significativa em mulheres que praticam musculação.

No estudo conduzido por Leal, et. al (2018), foi avaliada a prevalência da perda urinária involuntária e padrão miccional de praticantes de Crossfit, em mulheres que tiveram parto 
vaginal $(7,9 \%)$, cesária $(13,2 \%)$ e nulíparas $(78,9 \%)$, apenas 7,9\% dessa amostra apresentaram baixa prevalência da IU. Em outro estudo com mulheres praticantes dessa mesma modalidade, avaliou a IU associada ao estresse, os resultados sugerem uma possibilidade etiológica sem, no entanto, poder afirmar o tipo de incontinência (BOGEA, et al, 2019). Outro estudo que investigou a IU em mulheres praticantes de Crossfit conduzido por Ferreira et. al (2019), demonstrou a necessidade de estudos com amostras maiores com a possibilidade de busca por uma homogeneidade de treinos e avaliação.

O estudo de Almeida e Machado (2012), buscou identificar a prevalência da IU em mulheres praticantes de jump. Foram avaliadas mulheres multíparas e nulíparas e em outros momentos, indicando que a IU pode estar relacionada com prática, porém aponta a necessidade de maiores estudos sobre a temática com amostras maiores.

Hagovska, et al., (2018) indicam que os profissionais de saúde, em especial os fisioterapeutas, devem realizar prevenção através da informação com esportistas sobre os fatores de risco para o surgimento da IUE, e também implementar a fisioterapia preventiva para o fortalecimento da musculatura do assoalho pélvico.

As limitações do estudo referem-se à amostra, visto que foram incluídos apenas artigos disponíveis on-line gratuitamente e com nível de evidência II, que são evidências obtidas em estudos individuais com delineamento experimental, sem a presença de estudos dos níveis I, III, IV e V. Outra limitação foi na ausência de utilização de um instrumento para análise da qualidade dos artigos e busca dos artigos ter sido feita por um único pesquisador.

\section{Conclusão}

Essa revisão integrativa permitiu um conhecimento sobre a incontinência urinária que pode ser causada pela prática de esportes de alto impacto em mulheres. Porém indicou que nem sempre a IU está diretamente relacionada com a prática esportiva de alto impacto, e que a presença de outros fatores pode fazer emergir a incontinência urinária.

Dessa revisão emergem duas necessidades: a) estudos ampliados que possam aprofundar essa temática com maiores amostras e maior delineamento que permitam a identificação dos fatores que possa desencadear a incontinência e o seu tipo, bem como sua correlação com a modalidade esportiva, b) estudos randomizados que venham testar métodos de controle, conduzidos por fisioterapeutas, para incontinência urinária em atletas para possam manter a prática esportiva sem comprometimento de sua qualidade de vida. 
A Fisioterapia dispõe de informações que podem ser disseminadas para mulheres em espaços de saúde pública como forma de prevenção e ainda de recursos e técnicas que são viáveis no tratamento e controle da incontinência urinária, pois muitas mulheres não tem acesso por desconhecimento.

\section{Referências}

ALMEIDA, Priscilla Pereira de; MACHADO, Lívia Raquel Gomes. A prevalência de incontinência urinária em mulheres praticantes de jump. Fisioter. mov., Curitiba, v. 25, n. 1, p. 55-65, Mar. 2012. Available from <http://www.scielo.br/scielo.php?script=sci_ arttext\&pid=S0103-51502012000100006\&lng=en\&nrm=iso>. access on 02 Nov. 2020. https://doi.org/10.1590/S0103-51502012000100006.

ALVES, J. O. et al. Urinary Incontinence in Physically Active Young Women: Prevalence and Related Factors. International Journal of Sports Medicine, p. 937-941,2017.

AMORIM, Líllian Freire de; SARAIVA, Daiane Santos Dias; CIRQUEIRA, Rosana Porto. Prevalência de Incontinência Urinária em Mulheres Praticantes de Pilates e de Musculação. Id on Line Rev.Mult. Psic., Dezembro/2019, vol.13, n.48, p. 311-322. ISSN: 1981-1179.

ARAUJO, M. P. et al. Relação entre incontinência urinária em mulheres atletas corredoras de longa distância e distúrbio alimentar. RevAssocMedBras, São Paulo, p. 9-146, 2008.

BARRETO, E. et al. Sintomas do trato urinário inferior em mulheres que praticam exercício físico regularmente. Manual Therapy, Posturology \& Rehabilitation Journal, São Paulo, p. 773-791, ago./dez. 2014.

$\mathrm{BO}, \mathrm{K}$. et al. Are former female elite athletes more likely to experience urinary incontinence later in life than nonathletes? Journal of Medicine \& Science in Sports, Scandinavian, p. 100-104, 2010.

BOGÉA, Monique; GOMES, Priscila; DINUCCI, Ana; DUTRA, Fábio e ZAIDAN, Patrícia, 2018. "Incontinência urinária de esforço em mulheres praticantes de crossfit ${ }^{\circledR}$ : um estudo transversal de prevalência", International Journal of Development Research, 8, (07), 2164221645.

DA SILVA, Leidiany Bueno et al. Disfunções urinárias em mulheres praticantes de atividade física em academias - um estudo transversal. Revista Pesquisa em Fisioterapia, [S.1.], v. 8, n. 1, p. 71-78, set. 2018. ISSN 2238-2704. Disponível em: <https://www5.bahiana.edu.br/index.php/fisioterapia/article/view/1756>. Acesso em: 02 nov. 2020. doi:http://dx.doi.org/10.17267/2238-2704rpf.v8i1.1756.

FERRAZ FR, Ferraz AA, Nunes AFC, Latorre GRF. Prevalência de incontinência urinária em atletas praticantes de corrida de rua. Rev. Aten. Saúde. 2018;16(57):37-44. 
FERREIRA, Letícia; MELO, Vitória Laura de, DIAS, Mirella. Prevalência de incontinência urinária de esforço em mulheres praticantes de crossfit. Fisioterapia - Pedra Branca, 2019. Disponível em: http://www.riuni.unisul.br/handle/12345/8250, acesso 20 set. 2020.

FERNANDES, Susana Fernandes; COUTINHO, Emília Carvalho; DUARTE, João Carvalho; NELAS, Paula Alexandra Batista; CHAVES, Claudia Margarida Correia Balula Chaves; AMARAL, Odete. Qualidade de vida em mulheres com Incontinência Urinária. Revista de Enfermagem Referência - Série IV - n. ${ }^{\circ} 5$ - abr./mai./jun. 2015.

GRAM, M., \& B $\emptyset$, K. High level rhythmic gymnasts and urinary incontinence: Prevalence, risk factors, and influence on performance. Scandinavian journal of medicine \& science in sports, 30(1), 159-165, 2020. https://doi.org/10.1111/sms.13548

HAGOVSKA, M., Švihra, J., Buková, A., Dračková, D., \& Švihrová, V. (2018). Prevalence and risk of sport types to stress urinary incontinence in sportswomen: A cross-sectional study. Neurourology and urodynamics, 37(6), 1957-1964. https://doi.org/10.1002/nau.23538

HAVGOSKA, M. et al. Prevalence of Urinary Incontinence in Females Performing HighImpact Exercises. International Journal of Sports Medicine, República Eslovaca, p.210-216, 2017.

HAGOVSKA, M., et al. Prevalence and risk of sport types to stress urinary incontinence in sportswomen: A cross-sectional study. Neurourology and urodynamics, 37(6), 19571964, 2018. https://doi.org/10.1002/nau.23538

LEAL, Larissa de Oliveira et al. Prevalência de incontinência urinária e perfil miccional de mulheres praticantes de Crossfit ${ }^{\circledR}$. Fisioterapia Brasil, [S.1.], v. 21, n. 2, p. 197-203, maio 2020. ISSN 2526-9747. Disponível em: <http://www.portalatlanticaeditora.com.br/ index.php/fisioterapiabrasil/article/view/3621>. Acesso em: 02 nov. 2020. doi:http://dx.doi.org/10.33233/fb.v21i2.3621.

MACHADO, Lisandra da Silva. Avaliação funcional do assoalho pélvico em atletas e sua relação com a incontinência urinária. Dissertação (Pós- graduação em ciência da reabilitação) - Universidade Federal de Ciências da Saúde de Porto Alegre - UFCSPA, 2017.

NYGAARD, I.E.; SHAW, J.M. Physical activity and the pelvic floor. Am J Obstet Gynecol. v. 214, n. 2, p.164-71, 2016.

PATRIZZI, L J; VIANA, D A; SILVA, L M A; PEGORARI, M S. Incontinência urinária em mulheres jovens praticantes de exercício físico. R. Bras. Ci. e Mov. 2014; 22(3): 105-110

PENSIN, L.A., PEREIRA, G., FRANSOZI, J.D., PENSIN, L., BELLO, S.L., \& BRUM, S.P. Prevalência de incontinência urinária em mulheres do curso de educação física da Universidade do Sul de Santa Catarina, campus Tubarão. Rev. Arq. Catarin. Med. 2012; 41(4): 42-46.

PEREIRA, E.F. et al. Qualidade de vida: abordagens, conceitos e avaliação. Rev. bras. Educ. Fís. Esporte, São Paulo, v.26, n.2, p.241-50, abr./jun. 2012. 
PRIGOL, Sandra. SEBBEN, Vanessa. GUEDES, Janesca Mansur. Prevalência da incontinência urinária em mulheres praticantes de atividade física nas academias da cidade de Erechim, PERSPECTIVA, Erechim. v. 38, n.141, p. 121-130, março/2014.

ROSA, Patrícia Viana da; VARGAS, Patrícia Araújo de; KELLER, Kalina Durigon; LIMA, Caroline Helena Lazarotto de; KLAHR, Patrícia da Silva.; ROSA, Luis Henrique Telles da. Prevalência de incontinência urinária em mulheres praticantes de jump. Fisioterapia Brasil, v. 17, n. 1, p. 46-49, 2016.

SANTOS. Aline de Souza. A incontinência urinária e a prática da atividade fisica. Monografia apresentada como requisito parcial para a conclusão do Curso de Especialização em Fisiologia do Exercício, Setor de Ciências Biológicas, Universidade Federal do Paraná. CURITIBA, 2018.

TEIXEIRA, Elizabeth; MEDEIROS, Horácio Pires; NASCIMENTO, Marcia Helena Machado; SILVA, Bruna Alessandra Costa; RODRIGUES, Camila. Revisão Integrativa da Literatura passo-a-passo \& convergências com outros métodos de revisão. Rev Enferm UFPI, Teresina, 2(spe):3-7, dec., 2013.

\section{Como citar este artigo (Formato ABNT):}

SILVA, Zenilda Santos ; PALLES, Lucimauro Silva; FERREIRA, Juliana Barros. Prevalência da Incontinência Urinária em Mulheres Atletas Praticantes de Esporte de Alto Impacto: Uma Revisão Integrativa. Id on Line Rev.Mult. Psic., Dezembro/2020, vol.14, n.53, p. 543-555. ISSN: 1981-1179.

Recebido: 27/11/2020;

Aceito: $14 / 12 / 2020$. 